\title{
Simulation model for the study of possible consequences in the occurrence of a fire in various types of buildings
}

\author{
Mikhail Shahramanyan ${ }^{1}$, Issam Samara ${ }^{2}$, Ilya Sadrtdinov², Igor Malcev² \\ ${ }^{1}$ All-Russian Scientific Research Institute of Fire Protection EMERCOM of Russia \\ ${ }^{2}$ Don State Technical University, Institute of Service and Business (Branch) DSTU in Shakhty, \\ Russia
}

\begin{abstract}
This paper, describes a developed simulation model that gives the ability to simulate various situations of possible fire occurrence and study the behavior of people during their evacuation, taking into account various factors in different types of buildings, which allows the prediction of the consequences of a fire and subsequently develop rules for human behavior in extreme situations. Studies have shown that the optimization of movement during the simulation of a fire made it possible to reduce the likelihood of fire by approximately $20 \%$, and the presence of an emergency exit reduces it by another $30 \%$, which makes it possible to optimize the results of modeling the consequences of a fire in rooms of various types.
\end{abstract}

\section{Introduction}

Nowadays in the Russian Federation, great attention is paid to the issues of fire safety of the population, especially when they are found in various retail buildings or during cultural events. An example of this is the Decree of the Government of the Russian Federation of September 16, 2020 №1479 « About the approval of the Rules of the fire-fighting regime in the Russian Federation» [1]. This resolution considers in sufficient detail the issues and actions of both the management stuff and visitors in retail building, buildings during cultural events, etc. However, it must be kept in mind that the organization of fire supervision is mainly based on the periodic inspection of the object by fire supervision inspectors, followed by the issuance of instructions to eliminate the identified violations. The frequency of such inspections to assess the level of fire safety depends on the risk category of the object under examination and ranges from 3 to 10 years.

The disadvantages of this approach are: firstly, there is a significant time interval between inspections, and secondly, in the interval between inspections, there may be violations of the object operation or changes in its design, thirdly, due to the above; the probability of a major fire with possible loss of life and great economic damage may increase. In addition, it is difficult to describe in the instructions all possible situations that may happen during a fire, and even more so the process of its course and consequences. This is due to the fact that it is impossible to simulate all possible situations during training and fire safety exercises [2-6]. In this regard, it is very important to develop a complex of 
programs, that gives the ability to simulate and play any possible situations when a fire occurs and proceeds.

This work is devoted to the description of a developed simulation model, which makes it possible to simulate almost any situation that may arise when people are in various retail buildings or during cultural events. This simulation model can also be used to simulate the occurrence of a fire in almost any room, including offices, warehouses and other types of buildings.

\section{Methods and materials}

Let's assume that we are given a certain room that has one entrance. There is no emergency exit, the room is free, i.e. there are no obstacles in the room like tables, chairs and other possible obstacles. In this room, people constantly enter and exit, who evenly distributed throughout the room. At some point in time, a fire occurs at some point. All the people in the room rush to the exit. It is necessary to determine the degree of damage and the likelihood of unfavorable outcomes in the occurrence of a fire at an arbitrary point in the space of the room [7-12]. We will consider this statement of the problem as the initial and most simple one. In the future, a more complicated scenario will be studied in order to analyze all possible situations, such as the presence of obstacles, the presence of an emergency exit (possibly several), the presence of several sources of fire, the area of the room that is affected by the fire, etc.

To solve this problem, we will set a certain room, the size of which can be arbitrary, depending on the performance ЭBM, for example, let's assume the size of 20X20. The entrance door is one, i.e. the entrance and exit are carried out through it. So, there is no spare door. This door is consistently entered by people who can immediately exit or move around the room according to a uniform law, i.e. the probability of movement at any point in any direction is exactly the same, as shown in Figure 1. It should be noted that the law of movement of people around the room can be arbitrary, which is provided by the algorithm of the developed program.

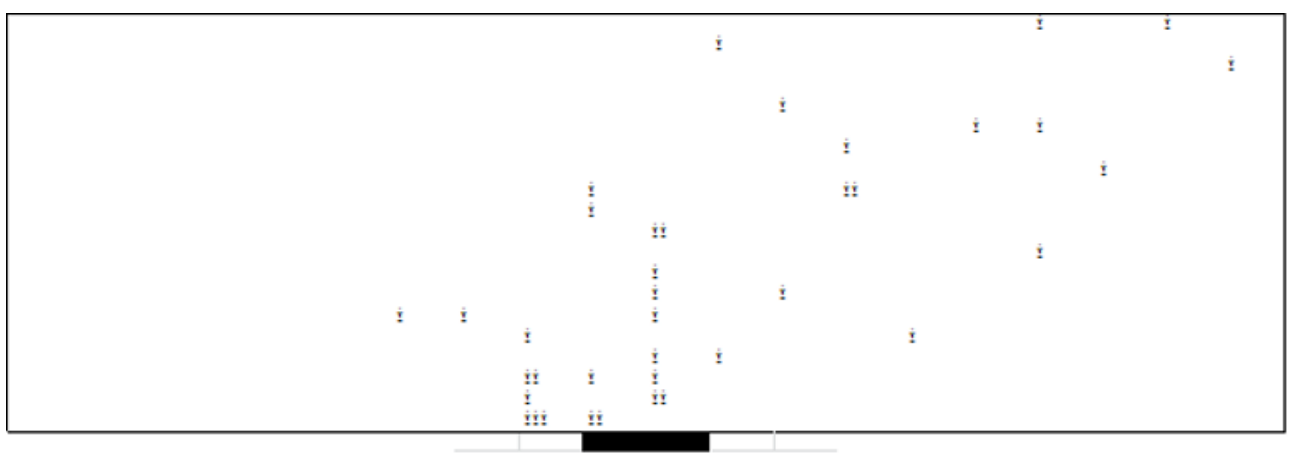

Fig. 1. The model of the room and the movement of people (entering -50 people, exiting -8 people)

From the room shown in Figure 1, it can be seen that out of 50 who entered, 8 people left, and the rest are evenly distributed throughout the room. The number of people entering the room can be set arbitrarily, however, take into account that for each room the number of people who are simultaneously in the room is regulated, with a threshold of $70-80 \%$ on the right, the cell «Number of people in the room» highlighted in yellow (if less highlighted in green). If the allowable value of people for a given room is exceeded, the cell «Number of people in the room» will be highlighted in red and, if desired, the number of incoming 
people can be suspended, i.e. entering the room is not allowed anymore, exiting is allowed as long as their number decreases below the threshold. This model gives the ability to check the number of people in the room constantly.

Further development of the model is to include at some point in time (set a priori upon reaching a certain number of people in the room) the coordinates of the occurrence of the fire which is set arbitrarily, the program itself asks the user about it. After entering the coordinates, this cell is colored red, as shown in Figure 2.

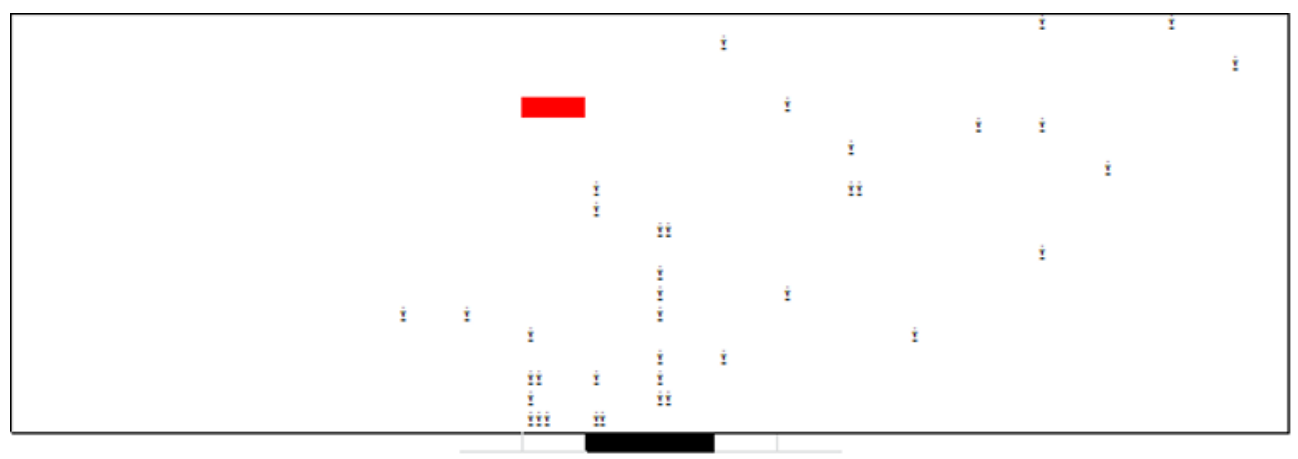

Fig. 2. The occurrence of a fire in the room (the source of the fire is highlighted in red)

After a fire starts, entering is prohibited, the door is available only for exiting. At the same time, how many people inside the room is constantly recorded, how many come out, how many died (got into fire), how many people near the fire source. After a while, the evacuation of people begins, i.e. everyone in the room begins to move towards the exit in an optimal way, considering that path should not pass near the source of the fire. Each person in the room has his own path, which can change as he approaches the exit. As soon as that the number of people at the exit exceeds the number that can exit through this door, the cell lights up «Crash has happened» and begins to calculate the number of people who may be affected when it occurs. Approximate trajectories of movement of people are shown in Figure 3 from different points of the room (left side). It should be noted that the trajectory for each person is calculated strictly individually and depends on a large number of factors.

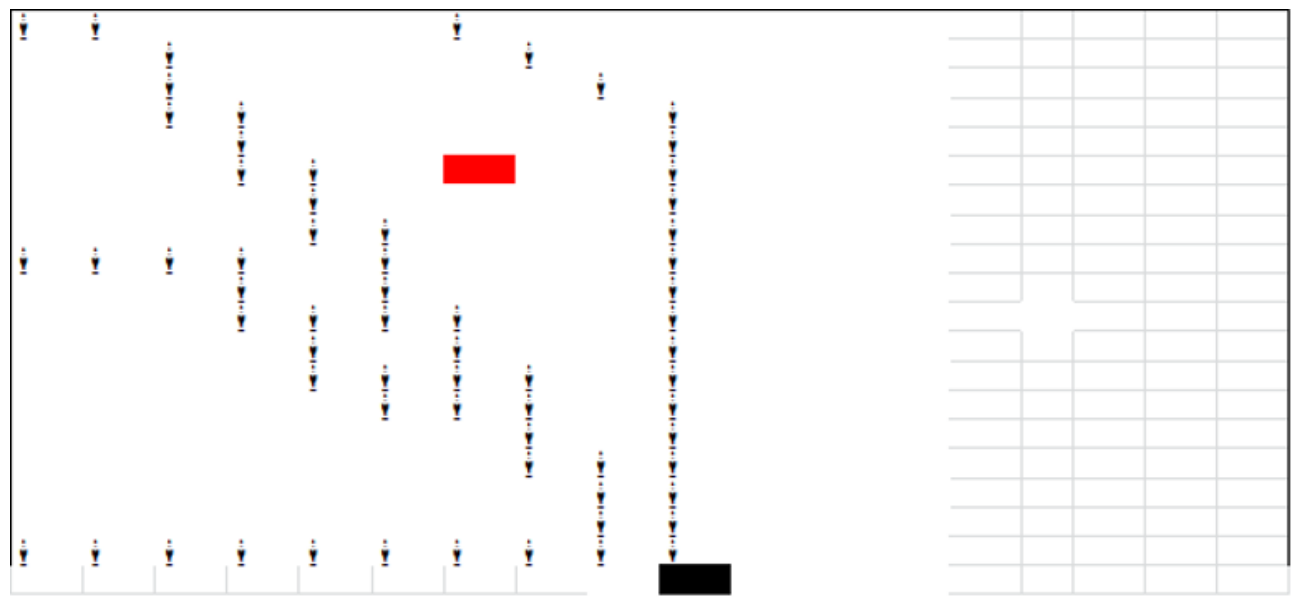

Fig. 3. The trajectory of movement of people in case of a fire occurrence 
Figure 3 shows that the trajectory of the movement is calculated relative to the point of occurrence of the fire. Optimization of the movement of people to the exit is provided at each step, alongside with the shortest route of movement ensuring not to bypass through fire.

\section{Results and discussion}

This software package gives the ability to simulate almost any situation and can be used as a simulator for considering various cases, as well as it serves as a simulation model for the formation of fire safety requirements for owners of trade and cultural and entertainment establishments.

\section{Conclusion}

1. On the basis of the conducted studies of the developed simulation model, the possibility of considering different situations in the occurrence of a fire in different types of buildings was shown.

2. During the evacuation of people in the simulation model, it is possible to provide an estimation of the dead, injured people, depending on the simulated situation.

3. Studies have shown that the optimization of movement during the simulation of a fire made it possible to reduce the likelihood of fire by approximately $20 \%$, and the presence of an emergency exit reduces it by another 30\%, which makes it possible to optimize the results of modeling the consequences of a fire in rooms of various types.

4. The simulation model is highly versatile; gives the ability to create a model of a room with any density of interfering objects, any number of entrance and emergency exits, points or sources of fire and simulate the evacuation process under any interfering factors.

\section{References}

1. State report «On the state of protection of the population and territories of the Russian Federation from natural and man-made emergencies in 2019», Moscow: EMERCOM of Russia (2020)

2. M.A. Shahramanyan. «Hardware and software complex for assessing the level of fire safety of an object in real time». Certificate of the National Register of Intellectual Property, 860-600-303 (2019)

3. M. Shahramanyan, V.I. Marchuk, I. Shrayfel, D. Chernyshov, B. Meskhi, O. Ananova. IOP Conf. Series: Materials Science and Engineering, 680, 1, 012026 (2019)

4. Ü. Çakiroğlu, S. Gökoğlu. Computers \& Education, 133, 56-68 (2019)

5. I. Muller. Heuristic methods in engineering development. Moscow: Radio and communication (1984)

6. K. Zhang, J. Suo, J. Chen, X. Liu, L. Gao. FedCSIS, 1297-1300 (2017)

7. I.O. Nikishin, I. Samara, V.I. Marchuk, Development of a people counting device in the indoor, in Proceedings of the VI National Scientific Conference of Students, Postgraduates and Young Scientists "Scientific Spring-2021", 17-21 May 2021, Shakhty, Russia (2021)

8. C. Maluk, M. Woodrow, J.L. Torero. Fire safety journal, 88, 104-112 (2017) 
9. K.J. Spyrou, I.A. Koromila. Reliability Engineering \& System Safety, 200, 106937 (2020)

10. R. Yang, F. Khan, M. Yang, D. Kong, C. Xu. Ocean Engineering, 157, 219-233 (2018)

11. J. Gehandler. Fire safety journal, 91, 973-981 (2017)

12. S.S. Valeev, N.V. Kondratyev, A.F. Yangirova. Bulletin USATU, 18, 1(62), 161-166 (2014) 